\title{
Bose-Einstein Correlations in the Hadronic Decays of the $\mathrm{Z}^{o}$
}

\author{
DELPHI Collaboration
}

\begin{abstract}
Bose-Einstein correlations between pairs of like sign charged particles produced in $e^{+} e^{-}$annihilations near the $\mathrm{Z}^{0}$ peak have been studied using data taken with the DELPHI detector at LEP. An enhancement is found in the production of pairs of identical pions of similar momenta, with respect to a reference sample. Under the hypothesis that the pions are emitted by a spherically symmetrical source with Gaussian density, the data indicate a radius of the source of $r=$ $0.62 \pm 0.04($ stat $) \pm 0.20($ syst $) \mathrm{fm}$. The large systematic uncertainty reflects the sensitivity of $r$ to the choice of the reference sample.
\end{abstract}


P.Abreu ${ }^{19}$, W.Adam ${ }^{45}$, T.Adye ${ }^{34}$, E.Agasi ${ }^{28}$, G.D.Alekseev ${ }^{13}$, P.Allen ${ }^{44}$, S.Almehed ${ }^{22}$, S.J.Alvsvaag ${ }^{4}$, U.Amaldi $^{7}$, E.G.Anassontzis ${ }^{3}$, A.Andreazza ${ }^{26}$, P.Antilogus ${ }^{23}$, W-D.Apel ${ }^{14}$, R.J.Apsimon ${ }^{34}$, B.Asman ${ }^{40}$, J-E.Augustin ${ }^{17}$, A.Augustinus ${ }^{28}$, P.Baillon ${ }^{7}, \quad$ P.Bambade ${ }^{17}, \quad$ F.Barao ${ }^{19}, \quad$ R.Barate ${ }^{11}$, G.Barbiellini ${ }^{42}$, D.Y.Bardin ${ }^{13}$, A.Baroncelli ${ }^{37}$, O.Barring ${ }^{22}$, J.A.Barrio ${ }^{24}, \quad$ W.Bartl ${ }^{45}, \quad$ M.J.Bates ${ }^{31}$, M.Battaglia $^{26}$, M.Baubillier $^{21}$, K-H.Becks ${ }^{47}$, C.J.Beeston ${ }^{31}$, M.Begalli ${ }^{33}$, P.Beilliere ${ }^{6}$, Yu.Belokopytov ${ }^{39}$, P.Beltran ${ }^{9}$, D.Benedic $^{8}$, M.Berggren ${ }^{17}$, D.Bertrand ${ }^{2}$, F.Bianchi ${ }^{41}$, M.S.Bilenky ${ }^{13}$, P.Billoir ${ }^{21}$, J.Bjarne ${ }^{22}$, D.Bloch ${ }^{8}$, S.Blyth $^{31}$, V.Bocci ${ }^{35}$, P.N.Bogolubov ${ }^{13}$, T.Bolognese ${ }^{36}$, M.Bonesini ${ }^{26}$, W.Bonivento ${ }^{26}$, P.S.L.Booth ${ }^{20}$, P.Borgeaud $^{36}$, G.Borisov ${ }^{39}$, H.Borner ${ }^{7}$, C.Bosio ${ }^{37}$, B.Bostjancic ${ }^{7}$, S.Bosworth ${ }^{31}$, O.Botner ${ }^{43}$, B.Bouquet ${ }^{17}$, C.Bourdarios $^{17}$, T.J.V.Bowcock ${ }^{20}$, M.Bozzo ${ }^{10}$, S.Braibant ${ }^{2}$, P.Branchini ${ }^{37}$, K.D.Brand ${ }^{32}$, R.A.Brenner ${ }^{7}$, H.Briand ${ }^{21}$, C.Bricman ${ }^{2}$, R.C.A.Brown ${ }^{7}$, N.Brummer ${ }^{28}$, J-M.Brunet ${ }^{6}$, L.Bugge ${ }^{30}$, T.Buran ${ }^{30}$, H.Burmeister ${ }^{7}$, J.A.M.A.Buytaert ${ }^{2}$, M.Caccia ${ }^{7}$, M.Calvi ${ }^{26}$, A.J.Camacho Rozas ${ }^{38}$, T.Camporesi ${ }^{7}$, V.Canale ${ }^{35}$, F.Cao $^{2}$, F.Carena ${ }^{7}$, L.Carroll ${ }^{20}$, C.Caso ${ }^{10}$, E.Castelli ${ }^{42}$, M.V.Castillo Gimenez ${ }^{44}$, A.Cattai ${ }^{7}$, F.R.Cavallo ${ }^{5}$, L.Cerrito $^{35}$, V.Chabaud ${ }^{7}$, A.Chan ${ }^{1}$, M.Chapkin ${ }^{39}$, Ph.Charpentier ${ }^{7}$, L.Chaussard ${ }^{17}$, J.Chauveau ${ }^{21}$, P.Checchia ${ }^{32}$, G.A.Chelkov $^{13}$, L.Chevalier $^{36}$, P.Chliapnikov ${ }^{39}$, V.Chorowicz ${ }^{21}$, J.T.M.Chrin ${ }^{44}$, R.Cirio ${ }^{41}$, M.P.Clara ${ }^{41}$, P.Collins $^{31}$, J.L.Contreras ${ }^{24}$, R.Contri ${ }^{10}$, E.Cortina ${ }^{44}$, G.Cosme ${ }^{17}$, F.Couchot ${ }^{17}$, H.B.Crawley ${ }^{1}$, D.Crennell ${ }^{34}$, G.Crosetti $^{10}$, M.Crozon ${ }^{6}$, J.Cuevas Maestro ${ }^{38}$, S.Czellar ${ }^{12}$, S.Dagoret ${ }^{17}$, E.Dahl-Jensen ${ }^{27}$, B.Dalmagne ${ }^{17}$, M.Dam $^{30}$, G.Damgaard ${ }^{27}$, G.Darbo ${ }^{10}$, E.Daubie ${ }^{2}$, A.Daum ${ }^{14}$, P.D.Dauncey ${ }^{31}$, M.Davenport ${ }^{7}$, P.David ${ }^{21}$, W.Da Silva ${ }^{21}$, C.Defoix ${ }^{6}$, D.Delikaris ${ }^{7}$, B.A.Della Riccia ${ }^{41}$, S.Delorme ${ }^{7}$, P.Delpierre ${ }^{6}$, N.Demaria ${ }^{41}$, A.De Angelis ${ }^{42}$, M.De Beer ${ }^{36}$, H.De Boeck ${ }^{2}$, W.De Boer ${ }^{14}$, C.De Clercq ${ }^{2}$, M.D.M.De Fez Laso ${ }^{44}$, N.De Groot ${ }^{28}$, C.De La Vaissiere ${ }^{21}$, B.De Lotto ${ }^{42}$, A.De Min ${ }^{26}$, H.Dijkstra ${ }^{7}$, L.Di Ciaccio ${ }^{35}$, F.Djama ${ }^{8}$, J.Dolbeau ${ }^{6}$, M.Donszelmann ${ }^{7}$, K.Doroba ${ }^{46}$, M.Dracos ${ }^{7}$, J.Drees ${ }^{47}$, M.Dris ${ }^{29}$, Y.Dufour ${ }^{6}$, L-O.Eek ${ }^{43}$, P.A.-M.Eerola ${ }^{7}$, R.Ehret $^{14}$, T.Ekelof ${ }^{43}$, G.Ekspong ${ }^{40}$, A.Elliot Peisert ${ }^{32}$, J-P.Engel ${ }^{8}$, D.Fassouliotis ${ }^{29}$, T.A.Fearnley ${ }^{4}$, M.Feindt $^{7}$, M.Fernandez Alonso ${ }^{38}$, A.Ferrer ${ }^{44}$, T.A.Filippas ${ }^{29}$, A.Firestone ${ }^{1}$, H.Foeth ${ }^{7}$, E.Fokitis ${ }^{29}$, F.Fontanelli ${ }^{10}$, K.A.J.Forbes ${ }^{20}$, B.Franek ${ }^{34}$, P.Frenkiel ${ }^{6}$, D.C.Fries ${ }^{14}$, A.G.Frodesen ${ }^{4}$, R.Fruhwirth ${ }^{45}$, F.Fulda-Quenzer ${ }^{17}$, K.Furnival $^{20}$, H.Furstenau ${ }^{14}$, J.Fuster ${ }^{7}$, G.Galeazzi ${ }^{32}$, D.Gamba ${ }^{41}$, C.Garcia ${ }^{44}$, J.Garcia ${ }^{38}$, C.Gaspar ${ }^{7}$, U.Gasparini $^{32}$, Ph.Gavillet ${ }^{7}$, E.N.Gazis ${ }^{29}$, J-P.Gerber ${ }^{8}$, P.Giacomelli ${ }^{7}$, R.Gokieli ${ }^{46}$, V.M.Golovatyuk ${ }^{13}$, J.J.Gomez Y Cadenas ${ }^{7}$, A.Goobar ${ }^{40}$, G.Gopal ${ }^{34}$, M.Gorski ${ }^{46}$, V.Gracco ${ }^{10}$, A.Grant ${ }^{7}$, F.Grard $^{2}$, E.Graziani ${ }^{37}$, G.Grosdidier $^{17}$, E.Gross ${ }^{7}$, P.Grosse-Wiesmann ${ }^{7}$, B.Grossetete ${ }^{21}$, J.Guy ${ }^{34}$, U.Haedinger ${ }^{14}$, F.Hahn ${ }^{7}$, M.Hahn $^{14}$, S.Haider $^{28}$, Z.Hajduk ${ }^{15}$, A.Hakansson ${ }^{22}$, A.Hallgren ${ }^{43}$, K.Hamacher ${ }^{47}$, G.Hamel De Monchenault ${ }^{36}$, W.Hao ${ }^{28}$, F.J.Harris ${ }^{31}$, T.Henkes ${ }^{7}$, J.J.Hernandez ${ }^{44}$, P.Herquet ${ }^{2}$, H.Herr ${ }^{7}$, T.L.Hessing ${ }^{20}$, I.Hietanen ${ }^{12}$, C.O.Higgins ${ }^{20}$, E.Higon $^{44}$, H.J.Hilke ${ }^{7}$, S.D.Hodgson ${ }^{31}$, T.Hofmokl ${ }^{46}$, R.Holmes ${ }^{1}$, S-O.Holmgren ${ }^{40}$, D.Holthuizen ${ }^{28}$, P.F.Honore ${ }^{6}$, J.E.Hooper ${ }^{27}$, M.Houlden ${ }^{20}$, J.Hrubec ${ }^{45}$, P.O.Hulth ${ }^{40}$, K.Hultqvist ${ }^{40}$, P.Ioannou ${ }^{3}$, D.Isenhower ${ }^{7}$, P-S.Iversen ${ }^{4}$ ， J.N.Jackson ${ }^{20}$ ， P.Jalocha ${ }^{15}$ ， G.Jarlskog ${ }^{22}$ ， P.Jarry ${ }^{36}$ ， B.Jean-Marie ${ }^{17}$, E.K.Johansson ${ }^{40}$, D.Johnson $^{20}$, M.Jonker ${ }^{7}$, L.Jonsson ${ }^{22}$, P.Juillot ${ }^{8}$, G.Kalkanis ${ }^{3}$, G.Kalmus ${ }^{34}$, F.Kapusta ${ }^{21}$, M.Karlsson ${ }^{7}$, E.Karvelas $^{9}$, S.Katsanevas ${ }^{3}$, E.C.Katsoufis ${ }^{29}$, R.Keranen ${ }^{12}$, J.Kesteman ${ }^{2}$, B.A.Khomenko ${ }^{13}$, N.N.Khovanski ${ }^{13}$, B.King $^{20}$, N.J.Kjaer ${ }^{7}$, H.Klein ${ }^{7}$, W.Klempt ${ }^{7}$, A.Klovning ${ }^{4}$, P.Kluit ${ }^{28}$, J.H.Koehne ${ }^{14}$, B.Koene ${ }^{28}$, P.Kokkinias ${ }^{9}$, M.Kopf $^{14}$, K.Korcyl ${ }^{15}$, A.V.Korytov ${ }^{13}$, V.Kostioukhine ${ }^{39}$, C.Kourkoumelis ${ }^{3}$, O.Kouznetsov ${ }^{13}$, P.H.Kramer ${ }^{47}$, J.Krolikowski $^{46}$, I.Kronkvist ${ }^{22}$, J.Krstic ${ }^{31}$, U.Kruener-Marquis ${ }^{47}$, W.Krupinski ${ }^{15}$, K.Kulka ${ }^{43}$, K.Kurvinen ${ }^{12}$, C.Lacasta $^{44}$, C.Lambropoulos ${ }^{9}$, J.W.Lamsa ${ }^{1}$, L.Lanceri ${ }^{42}$, V.Lapin ${ }^{39}$, J-P.Laugier ${ }^{36}$, R.Lauhakangas ${ }^{12}$, G.Leder $^{45}$, F.Ledroit ${ }^{11}$, R.Leitner ${ }^{7}$, Y.Lemoigne ${ }^{36}$, J.Lemonne ${ }^{2}$, G.Lenzen ${ }^{47}$, V.Lepeltier ${ }^{17}$, A.Letessier-Selvon $^{21}$, J.M.Levy ${ }^{8}$, E.Lieb ${ }^{47}$, D.Liko ${ }^{45}$, E.Lillethun ${ }^{4}$, J.Lindgren ${ }^{12}$, R.Lindner ${ }^{47}$, A.Lipniacka ${ }^{46}$, I.Lippi $^{32}$, B.Loerstad ${ }^{22}$, M.Lokajicek ${ }^{13}$, J.G.Loken ${ }^{31}$, A.Lopez-Fernandez ${ }^{17}$, M.A.Lopez Aguera ${ }^{38}$, M.Los $^{28}$,

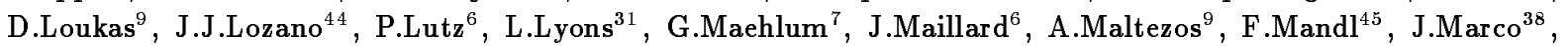
M.Margoni $^{32}$, J-C.Marin ${ }^{7}$, A.Markou ${ }^{9}$, T.Maron ${ }^{47}$, S.Marti ${ }^{44}$, L.Mathis ${ }^{1}$, F.Matorras ${ }^{38}$, C.Matteuzzi ${ }^{26}$, G.Matthiae $^{35}$ ， M.Mazzucato ${ }^{32}$ ， M.Mc Cubbin ${ }^{20}$ ， R.Mc Kay ${ }^{1}$ ， R.Mc Nulty ${ }^{20}$ ， G.Meola ${ }^{10}$, C.Meroni ${ }^{26}$, W.T.Meyer ${ }^{1}$, M.Michelotto ${ }^{32}$, I.Mikulec ${ }^{45}$, W.A.Mitaroff ${ }^{45}$, G.V.Mitselmakher ${ }^{13}$, U.Mjoernmark ${ }^{22}$, T.Moa $^{40}$, R.Moeller $^{27}$, K.Moenig ${ }^{7}$, M.R.Monge ${ }^{10}$, P.Morettini ${ }^{10}$, H.Mueller ${ }^{14}$, W.J.Murray ${ }^{34}$, B.Muryn ${ }^{17}$, G.Myatt $^{31}$, F.Naraghi $^{21}$, F.L.Navarria ${ }^{5}$, P.Negri ${ }^{26}$, B.S.Nielsen ${ }^{27}$, B.Nijjhar ${ }^{20}$, V.Nikolaenko ${ }^{39}$, P.E.S.Nilsen ${ }^{4}$, P.Niss ${ }^{40}$, V.Obraztsov $^{39}$, A.G.Olshevski ${ }^{13}$, R.Orava ${ }^{12}$, A.Ostankov ${ }^{39}$, K.Osterberg ${ }^{12}$, A.Ouraou ${ }^{36}$, M.Paganoni ${ }^{26}$, R.Pain $^{21}$, H.Palka ${ }^{28}$, Th.D.Papadopoulou ${ }^{29}$, L.Pape ${ }^{7}$, A.Passeri $^{37}$, M.Pegoraro ${ }^{32}$, J.Pennanen ${ }^{12}$, V.Perevozchikov ${ }^{39}$, M.Pernicka ${ }^{45}$, A.Perrotta ${ }^{5}$, A.Petrolini ${ }^{10}$, T.E.Pettersen ${ }^{32}$, F.Pierre ${ }^{36}$, M.Pimenta ${ }^{19}$, O.Pingot $^{2}$, M.E.Pol ${ }^{7}$, G.Polok ${ }^{15}$, P.Poropat ${ }^{42}$, P.Privitera ${ }^{14}$, A.Pullia ${ }^{26}$, D.Radojicic ${ }^{31}$, S.Ragazzi ${ }^{26}$, P.N.Ratoff ${ }^{18}$, A.L.Read ${ }^{30}$, N.G.Redaelli ${ }^{26}$, M.Regler ${ }^{45}$, D.Reid ${ }^{20}$, P.B.Renton ${ }^{31}$, L.K.Resvanis ${ }^{3}$, F.Richard ${ }^{17}$, M.Richardson ${ }^{20}$, J.Ridky ${ }^{13}$, G.Rinaudo ${ }^{41}$, I.Roditi ${ }^{16}$, A.Romero ${ }^{41}$, I.Roncagliolo ${ }^{10}$, P.Ronchese ${ }^{32}$, C.Ronnqvist $^{12}$, E.I.Rosenberg ${ }^{1}$, S.Rossi ${ }^{7}$, U.Rossi ${ }^{5}$, E.Rosso ${ }^{7}$, P.Roudeau ${ }^{17}$, T.Rovelli ${ }^{5}$, W.Ruckstuhl ${ }^{28}$, V.Ruhlmann ${ }^{36}$, A.Ruiz ${ }^{38}$, K.Rybicki ${ }^{15}$, H.Saarikko ${ }^{12}$, Y.Sacquin ${ }^{36}$, G.Sajot ${ }^{11}$, J.Salt ${ }^{44}$, J.Sanchez ${ }^{24}$, M.Sannino $^{10}$, S.Schael ${ }^{14}$, H.Schneider ${ }^{14}$, M.A.E.Schyns ${ }^{47}$, G.Sciolla ${ }^{41}$, F.Scuri ${ }^{42}$, A.M.Segar ${ }^{31}$, R.Sekulin ${ }^{34}$, M.Sessa ${ }^{42}$, G.Sette ${ }^{10}$, R.Seufert ${ }^{14}$, R.C.Shellard ${ }^{33}$, I.Siccama ${ }^{28}$, P.Siegrist ${ }^{36}$, S.Simonetti ${ }^{10}$, F.Simonetto ${ }^{32}$, 


\begin{abstract}
A.N.Sisakian ${ }^{13}$, T.B.Skaali ${ }^{30}$, G.Skjevling ${ }^{30}$, G.Smadja ${ }^{36,23}$, N.Smirnov ${ }^{39}$, G.R.Smith ${ }^{34}$, R.Sosnowski ${ }^{46}$, T.S.Spassoff ${ }^{11}$, E.Spiriti ${ }^{37}$, S.Squarcia ${ }^{10}$, H.Staeck ${ }^{47}$, C.Stanescu ${ }^{37}$, S.Stapnes ${ }^{30}$, G.Stavropoulos ${ }^{9}$, F.Stichelbaut ${ }^{2}$, A.Stocchi ${ }^{17}$, J.Strauss ${ }^{45}$, J.Straver $^{7}$, R.Strub ${ }^{8}$, M.Szczekowski $^{46}$, M.Szeptycka $^{46}$, P.Szymanski $^{46}$, T.Tabarelli $^{26}$, S.Tavernier ${ }^{2}$, O.Tchikilev ${ }^{39}$, G.E.Theodosiou ${ }^{9}$, A.Tilquin ${ }^{25}$, J.Timmermans ${ }^{28}$, V.G.Timofeev ${ }^{13}$, L.G.Tkatchev ${ }^{13}$, T.Todorov ${ }^{8}$, D.Z.Toet ${ }^{28}$, O.Toker ${ }^{12}$, A.Tomaradze ${ }^{39}$, E.Torassa ${ }^{41}$, L.Tortora ${ }^{37}$, M.T.Trainor ${ }^{31}$, D.Treille $^{7}$, U.Trevisan ${ }^{10}$, W.Trischuk ${ }^{7}$, G.Tristram ${ }^{6}$, C.Troncon ${ }^{26}$, A.Tsirou ${ }^{7}$, E.N.Tsyganov ${ }^{13}$, M.Turala ${ }^{15}$, M-L.Turluer ${ }^{36}$, T.Tuuva ${ }^{12}$, I.A.Tyapkin ${ }^{13}$, M.Tyndel $^{34}$, S.Tzamarias ${ }^{7}$, S.Ueberschaer ${ }^{47}$, O.Ullaland ${ }^{7}$, V.Uvarov $^{39}$, G.Valenti ${ }^{5}$, E.Vallazza ${ }^{41}$, J.A.Valls Ferrer ${ }^{44}$, C.Vander Velde ${ }^{2}$, G.W.Van Apeldoorn ${ }^{28}$, P.Van Dam $^{28}$, W.K.Van Doninck ${ }^{2}$, J.Varela ${ }^{19}$, P.Vaz ${ }^{7}$, G.Vegni ${ }^{26}$, L.Ventura ${ }^{32}$, W.Venus ${ }^{34}$, F.Verbeure ${ }^{2}$, L.S.Vertogradov $^{13}$, D.Vilanova ${ }^{36}$, L.Vitale ${ }^{12}$, E.Vlasov ${ }^{39}$, S.Vlassopoulos ${ }^{29}$, A.S.Vodopyanov ${ }^{13}$, M.Vollmer $^{47}$, S.Volponi $^{5}$, G.Voulgaris ${ }^{3}$, M.Voutilainen ${ }^{12}$, V.Vrba ${ }^{37}$, H.Wahlen ${ }^{47}$, C.Walck ${ }^{40}$, F.Waldner ${ }^{42}$, M.Wayne ${ }^{1}$, A.Wehr $^{47}$, M.Weierstall ${ }^{47}$, P.Weilhammer ${ }^{7}$, J.Werner ${ }^{47}$, A.M.Wetherell ${ }^{7}$, J.H.Wickens ${ }^{2}$, J.Wikne ${ }^{30}$, G.R.Wilkinson ${ }^{31}$, W.S.C.Williams ${ }^{31}$, M.Winter ${ }^{8}$, D.Wormald ${ }^{30}$, G.Wormser ${ }^{17}$, K.Woschnagg ${ }^{43}$, N.Yamdagni ${ }^{40}$, P.Yepes $^{7}$, A.Zaitsev ${ }^{39}$, A.Zalewska ${ }^{15}$, P.Zalewski ${ }^{17}$, D.Zavrtanik ${ }^{7}$, E.Zevgolatakos ${ }^{9}$, G.Zhang ${ }^{47}$, N.I.Zimin ${ }^{13}$, M.Zito $^{36}$, R.Zitoun ${ }^{21}$, R.Zuberi ${ }^{31}$, R.Zukanovich Funchal ${ }^{6}$, G.Zumerle ${ }^{32}$, J.Zuniga ${ }^{44}$
\end{abstract}

\footnotetext{
${ }^{1}$ Ames Laboratory and Department of Physics, Iowa State University, Ames IA 50011, USA

${ }^{2}$ Physics Department, Univ. Instelling Antwerpen, Universiteitsplein 1, B-2610 Wilrijk, Belgium and IIHE, ULB-VUB, Pleinlaan 2, B-1050 Brussels, Belgium

and Service de Phys. des Part. Elém., Faculté des Sciences, Université de l'Etat Mons, Av. Maistriau 19 , B-7000

Mons, Belgium

${ }^{3}$ Physics Laboratory, University of Athens, Solonos Str. 104, GR-10680 Athens, Greece

${ }^{4}$ Department of Physics, University of Bergen, Allégaten 55, N-5007 Bergen, Norway

${ }^{5}$ Dipartimento di Fisica, Università di Bologna and INFN, Via Irnerio 46, I-40126 Bologna, Italy

${ }^{6}$ Collège de France, Lab. de Physique Corpusculaire, 11 pl. M. Berthelot, F-75231 Paris Cedex 05, France

${ }^{7}$ CERN, CH-1211 Geneva 23, Switzerland

${ }^{8}$ Centre de Recherche Nucléaire, IN2P3 - CNRS/ULP - BP20, F-67037 Strasbourg Cedex, France

${ }^{9}$ Institute of Nuclear Physics, N.C.S.R. Demokritos, P.O. Box 60228, GR-15310 Athens, Greece

${ }^{10}$ Dipartimento di Fisica, Università di Genova and INFN, Via Dodecaneso 33, I-16146 Genova, Italy

${ }^{11}$ Institut des Sciences Nucléaires, Université de Grenoble 1, F-38026 Grenoble, France

${ }^{12}$ Research Institute for High Energy Physics, SEFT, Siltavuorenpenger 20 C, SF-00170 Helsinki, Finland

13 Joint Institute for Nuclear Research, Dubna, Head Post Office, P.O. Box 79, 101000 Moscow, USSR.

${ }^{14}$ Institut für Experimentelle Kernphysik, Universität Karlsruhe, Postfach 6980, D-7500 Karlsruhe 1, FRG

${ }^{15}$ High Energy Physics Laboratory, Institute of Nuclear Physics, Ul. Kawiory 26 a, PL-30055 Krakow 30, Poland

${ }^{16}$ Centro Brasileiro de Pesquisas, rua Xavier Sigaud 150, RJ-22290 Rio de Janeiro, Brazil

${ }^{17}$ Université de Paris-Sud, Lab. de l'Accélérateur Linéaire, Bat 200, F-91405 Orsay, France

${ }^{18}$ School of Physics and Materials, University of Lancaster - Lancaster LA1 4YB, UK

${ }^{19}$ LIP, Av. E. Garcia, 14 and Inst. Sup. Te'cnico, Univ. Te'cnica de Lisboa, Av. R. Pais, P-1000 Lisbon, Portugal

${ }^{20}$ Department of Physics, University of Liverpool, P.O. Box 147, GB - Liverpool L69 3BX, UK

${ }^{21}$ LPNHE, Universités Paris VI et VII, Tour 33 (RdC), 4 place Jussieu, F-75230 Paris Cedex 05, France

${ }^{22}$ Department of Physics, University of Lund, Sölvegatan 14, S-22363 Lund, Sweden

${ }^{23}$ Université Claude Bernard de Lyon, 43 Bd du 11 Novembre 1918, F-69622 Villeurbanne Cedex, France

${ }^{24}$ Universidad Complutense, Avda. Complutense s/n, E-28040 Madrid, Spain

${ }^{25}$ Univ. d'Aix - Marseille II - Case 907 - 70, route Léon Lachamp, F-13288 Marseille Cedex 09, France

${ }^{26}$ Dipartimento di Fisica, Università di Milano and INFN, Via Celoria 16, I-20133 Milan, Italy

${ }^{27}$ Niels Bohr Institute, Blegdamsvej 17, DK-2100 Copenhagen 0, Denmark

${ }^{28}$ NIKHEF-H, Postbus 41882, NL-1009 DB Amsterdam, The Netherlands

${ }^{29}$ National Technical University, Physics Department, Zografou Campus, GR-15773 Athens, Greece

${ }^{30}$ Physics Department, University of Oslo, Blindern, N-1000 Oslo 3, Norway

${ }^{31}$ Nuclear Physics Laboratory, University of Oxford, Keble Road, GB - Oxford OX1 3RH, UK

32 Dipartimento di Fisica, Università di Padova and INFN, Via Marzolo 8, I-35131 Padua, Italy

33 Depto. de Fisica, Pontificia Univ. Católica, C.P. 38071 RJ-22453 Rio de Janeiro, Brazil

34 Rutherford Appleton Laboratory, Chilton, GB - Didcot OX11 OQX, UK

${ }^{35}$ Dipartimento di Fisica, Università di Roma II and INFN, Tor Vergata, I-00173 Rome, Italy

${ }^{36}$ CEN-Saclay, DPhPE, F-91191 Gif-sur-Yvette Cedex, France

${ }^{37}$ Istituto Superiore di Sanità, Ist. Naz. di Fisica Nucl. (INFN), Viale Regina Elena 299, I-00161 Rome, Italy

${ }^{38}$ Facultad de Ciencias, Universidad de Santander, av. de los Castros, E - 39005 Santander, Spain

${ }^{39}$ Inst. for High Energy Physics, Serpukow P.O. Box 35, Protvino, (Moscow Region), USSR.

${ }^{40}$ Institute of Physics, University of Stockholm, Vanadisvägen 9, S-113 46 Stockholm, Sweden

${ }^{41}$ Dipartimento di Fisica Sperimentale, Università di Torino and INFN, Via P. Giuria 1, I-10125 Turin, Italy

${ }^{42}$ Dipartimento di Fisica, Università di Trieste and INFN, Via A. Valerio 2, I-34127 Trieste, Italy

and Istituto di Fisica, Università di Udine, I-33100 Udine, Italy

${ }^{43}$ Department of Radiation Sciences, University of Uppsala, P.O. Box 535, S-751 21 Uppsala, Sweden

${ }^{44}$ Inst. de Fisica Corpuscular IFIC, Centro Mixto Univ. de Valencia-CSIC, and Departamento de Fisica Atomica

Molecular y Nuclear, Univ. de Valencia, Avda. Dr. Moliner 50, E-46100 Burjassot (Valencia), Spain

${ }^{45}$ Institut für Hochenergiephysik, Österr. Akad. d. Wissensch., Nikolsdorfergasse 18, A-1050 Vienna, Austria

${ }^{46}$ Inst. Nuclear Studies and, University of Warsaw, U1. Hoza 69, PL-00681 Warsaw, Poland

${ }^{47}$ Fachbereich Physik, University of Wuppertal, Postfach 100 127, D-5600 Wuppertal 1, FRG
} 


\section{Introduction}

An enhancement in the production of pairs of pions of the same charge and similar momenta produced in high energy collisions was first observed in antiproton annihilations and attributed to Bose-Einstein statistics appropriate to identical pion pairs [1].

Bose-Einstein correlations between pion pairs can be used to study the space-time structure of the hadronization source [2]. This has been done for hadron-hadron, heavy ion, muon-hadron and $e^{+} e^{-}$collisions (see Ref. [3] for reviews, and Ref. [4] for results of $e^{+} e^{-}$experiments below the $\mathrm{Z}^{0}$ energy). Measurements at the LEP $e^{+} e^{-}$collider have been presented recently $[5,6]$, and disagree in the estimate of the strength of the correlation.

This letter describes an investigation of Bose-Einstein correlations in hadronic decays of the $\mathrm{Z}^{0}$ at a centre of mass energy of about $91 \mathrm{GeV}$, using data collected with the DELPHI detector [7] at LEP in 1991.

\section{Analysis}

To study the enhanced probability for emission of two identical bosons it is useful to define a correlation function $R$ :

$$
R\left(p_{1}, p_{2}\right)=\frac{P\left(p_{1}, p_{2}\right)}{P\left(p_{1}\right) P\left(p_{2}\right)}
$$

where $P\left(p_{1}, p_{2}\right)$ is the two-particle probability density, subject to Bose-Einstein symmetrization, and $P\left(p_{i}\right)$ is the corresponding single particle quantity for a particle with four-momentum $p_{i}$. In practice, $P\left(p_{1}\right) P\left(p_{2}\right)$ is often replaced by a reference 2 -particle distribution $P_{o}\left(p_{1}, p_{2}\right)$, which, ideally, resembles $P\left(p_{1}, p_{2}\right)$ in all respects, apart from the lack of Bose-Einstein symmetrization.

If $f(x)$ is the space-time distribution of the source, $R\left(p_{1}, p_{2}\right)$ takes the form

$$
R\left(p_{1}, p_{2}\right)=1+|G[f(x)]|^{2},
$$

where $G[f(x)]=\int f(x) e^{-\imath\left(p_{1}-p_{2}\right) \cdot x} d x$ is the Fourier transform of $f(x)$. Thus by studying the correlations between the momenta of pion pairs one can determine the distribution of the points of origin of the pions. The effect can be described in the variable $Q$, defined by $Q^{2}=M^{2}(\pi \pi)-4 m_{\pi}^{2}$, where $M$ is the invariant mass of the two pions.

The correlation function is often parametrized by the function:

$$
R(Q)=1+\lambda e^{-r^{2} Q^{2}}
$$

where the parameter $r$ gives the source size and $\lambda$ measures the strength of the correlation between the pions, being 0 for a completely coherent source and 1 for a completely incoherent one.

An expression slightly different from (1) is often used in the literature to analyze the experimental data:

$$
R(Q)=N(1+\delta \cdot Q)\left(1+\lambda e^{-r^{2} Q^{2}}\right)
$$

where the factor $(1+\delta \cdot Q)$ is introduced to take into account possible long-range momentum correlations in the form of a slow rise, and $N$ is a normalization factor.

The definition of an appropriate reference sample from which $P_{o}\left(p_{1}, p_{2}\right)$ can be obtained is the major problem of this kind of studies. In order to estimate systematic errors in the 
evaluation of the correlation function, two different reference samples have been used in the present study:

a. Pairs of oppositely charged particles have been taken.

b. An artificial sample of "mixed" particles has been obtained by dividing the event into two halves by a plane perpendicular to the thrust axis. The charged particles from one half were then combined with those from the other, reflecting through the origin the momentum of one particle in the pair.

This method requires a clear 2-jet topology, and thus it was applied only to events with a thrust value $T>0.95$.

\section{Experimental procedure and event selec- tion}

The sample of events used in the analysis was collected by the DELPHI detector at the LEP $e^{+} e^{-}$collider during 1991.

A description of the apparatus can be found in Ref. [7]. Features of the apparatus relevant for the analysis of multihadronic final states (with emphasis on the detection of charged particles) are outlined in Ref. [8]. The present analysis relies on the information provided by the central tracking detectors: the Micro Vertex Detector (VD), the Inner Detector (ID), the Time Projection Chamber (TPC), and the Outer Detector (OD).

The central tracking system of DELPHI covers the region between $25^{\circ}$ and $155^{\circ}$ in polar angle $\theta$, with reconstruction efficiency near to 1 . The average momentum resolution for the charged particles in hadronic final states is in the range $\Delta p / p \simeq 0.001 p$ to $0.01 p$ $(p$ in $\mathrm{GeV} / \mathrm{c}$ ), depending on which detectors are included in the track fit.

Only charged particles fulfilling the following criteria were used in this analysis: $(a)$ impact parameter at the primary vertex below $1 \mathrm{~cm}$ in radius from the beam axis and below $5 \mathrm{~cm}$ along the beam axis; (b) momentum, $p$, larger than $0.1 \mathrm{GeV} / \mathrm{c} ;(c)$ measured track length in the TPC above $50 \mathrm{~cm} ;(d)$ polar angle, $\theta$, between $25^{\circ}$ and $155^{\circ}$.

All these particles were assumed to be pions.

Hadronic events were then selected by requiring that $(\alpha)$ each of the two hemispheres $\cos \theta<0$ and $\cos \theta>0$ contained a total energy of the charged particles larger than 3 $\mathrm{GeV} ;(\beta)$ the total energy of the charged particles seen in both hemispheres together exceeded $15 \mathrm{GeV} ;(\gamma)$ there were at least 5 charged particles with momenta above 0.2 $\mathrm{GeV} / \mathrm{c} ;(\delta)$ the polar angle $\theta$ of the sphericity axis was in the range $40^{\circ}<\theta<140^{\circ}$ (this selection ensures that the retained events are well contained inside the TPC).

A total of 75,576 events satisfying these selections were used in the present analysis. Events due to beam-gas scattering and to $\gamma \gamma$ interactions have been estimated to be less than $0.1 \%$ of the sample; background from $\tau^{+} \tau^{-}$events was calculated to be less than $0.2 \%$.

Only events in which the thrust value was larger than 0.95 were used in the part of this analysis based on a "mixed" reference sample. The 34,984 events which passed this 
selection have a clear back to back two-jet topology, which ensures the correct functioning of the mixing technique.

Particles were paired only if each had a momentum below $5 \mathrm{GeV} / \mathrm{c}$, to avoid the limits of phase space where dynamical correlations are strong. Each particle was required to have a transverse momentum with respect to the beam axis above $250 \mathrm{MeV} / \mathrm{c}$, in order to exclude tracks curling inside the magnetic field, that was parallel to the beam axis. In addition, tracks were required to give a signal in the VD.

To exclude partially overlapping track pairs with potential problems in reconstruction, pairs were rejected if their opening angle was smaller than $2^{\circ}$. The cut $Q>60 \mathrm{MeV} / \mathrm{c}$ was imposed in addition, to reduce possible residual contaminations from photon conversions not correctly reproduced in the simulation, and the influence of correlated pions from $\eta^{\prime}$ decays.

The influence of detector effects and selections were studied by means of the detailed detector simulation Monte Carlo program DELSIM [9]. A sample of 75,000 events was generated using the JETSET 7.2 Parton Shower (PS) [10] Monte Carlo with parameters tuned as in [11] (without including any Bose-Einstein symmetrization), followed through the simulation of the detector, and processed through the same reconstruction and analysis chain as the real data.

\section{Results}

Figure 1 shows the distribution as a function of $Q$ of the numbers of like and unlike pion pairs found. In the unlike pairs, the presence of the pions coming from the decays of $K_{S}^{o}$ and $\rho^{o}$ is evident.

The ratio $R_{+-}(Q)=N_{ \pm \pm}(Q) / N_{+-}(Q)$, where $N_{ \pm \pm}$and $N_{+-}$are the number of like and unlike-charged pairs, is shown in Figure 2, together with the Monte Carlo distribution in which the Bose-Einstein effect was not simulated. The enhancement due to Bose-Einstein correlations is clearly visible in the region $Q<0.3 \mathrm{GeV} / \mathrm{c}$. The dips near $Q=0.4 \mathrm{GeV} / \mathrm{c}$ and $Q=0.7 \mathrm{GeV} / \mathrm{c}$ in both data and simulation are caused by contributions to the denominator by $K_{S}^{o}$ and $\rho^{o}$ decays.

In principle the bias due to residual correlations in the reference sample can be removed by dividing the ratio obtained from the data by the equivalent ratio from the simulation. This normalized ratio for the unlike charged reference sample is plotted in Figure 3a, with the fit of form (2) superimposed. The results of the fit are $r=0.83 \pm 0.03 \mathrm{fm}$, $\lambda=0.31 \pm 0.02\left(\chi 2=65 / 73\right.$ Degrees of Freedom, ${ }^{\dagger}$ correlation coefficient $\left.C(r \lambda)=0.19\right)$. Quantitative results from this correction procedure require a satisfactory model of non Bose-Einstein correlations in the simulation. In order to get an idea of the possible systematic error induced by bad modelling, the same analysis was done with the "mixed" reference sample described above.

First it was verified that:

\footnotetext{
${ }^{\dagger}$ The region from $\mathrm{Q}=0.4 \mathrm{GeV} / \mathrm{c}$ to $\mathrm{Q}=0.8 \mathrm{GeV} / \mathrm{c}$ (corresponding to $K^{0}$ and $\rho^{0}$ ), where the discrepancies between data and simulation are mainly concentrated, was removed from the fit.
} 
a. The procedure using unlike-sign pairs as a reference gives completely compatible results in the full event sample as in the subsample of 2 -jet events with thrust, $T$, above 0.95 .

b. The results using unlike-sign pairs as reference, in the subsample of 2 -jet events defined as in a., were not affected by the requirement that both particles in like and unlike pairs were in the same hemisphere relative to the thrust axis.

Then the "mixing" procedure was used in the subsample of 2 -jet events, with the result shown in Figure 3b. The fit to form (2) gives $r=0.47 \pm 0.03 \mathrm{fm}, \lambda=0.24 \pm 0.02$ $(\chi 2=98 / 93 \mathrm{DF}, C(r \lambda)=0.59)$. The results of the same technique using a stronger 2 -jet selection cut (thrust $T>0.97$ ), or by requiring the acollinearity between the axes of the two jets $\stackrel{\ddagger}{\ddagger}$ to be smaller than $3^{\circ}$, were consistent.

Two cuts described in Section 3 could in principle bias the effect measured, and the stability of the results was checked against dependence on these cuts.

1. The cut on the opening angle of the pairs $\left(2^{\circ}\right)$ was varied between $1^{\circ}$ and $5^{\circ}$;

2. The cut on the maximum impact parameter to the primary vertex in the plane perpendicular to the beam $(1 \mathrm{~cm})$ was varied between $0.5 \mathrm{~cm}$ and $2 \mathrm{~cm}$;

without significant changes in $r$ or $\lambda$.

Corrections are often made for two causes that could attenuate the effect of BoseEinstein correlations seen in Figure 3:

a. Particle identification was not used in this analysis, where all particles were assumed to be pions. The Bose-Einstein effect is thus attenuated by the presence of pairs of particles in which one is not a pion. Particle misidentification can be taken into account by subtracting from both the real density and the reference density distributions the fraction of pairs in which at least one particle is not a pion, as obtained from simulation. This fraction is found to be about $27 \%$ in average, smoothly growing from $15 \%$ at $Q=0.06 \mathrm{GeV} / \mathrm{c}$ to $30 \%$ at $Q=1 \mathrm{GeV} / \mathrm{c}$, and then reaching a plateau.

b. The final state Coulomb interaction modifies the two-pion relative momentum distribution in a different way for like $(l)$ and unlike $(u)$ pairs. The Coulomb effect is usually parametrized in the literature by the Gamow factors [12]

$$
W_{l}(\eta)=\frac{\left(e^{2 \pi \eta}-1\right)}{2 \pi \eta}, \quad W_{u}(\eta)=\frac{\left(1-e^{-2 \pi \eta}\right)}{2 \pi \eta}
$$

where $\eta=\alpha m_{\pi} / Q$. Data have been corrected for this final state interaction by weighting each like (unlike) pair with $W_{l}\left(W_{u}\right)$. Recent calculations [13] suggest that the Gamow factor overestimates the size of the final state Coulomb interaction; however it was decided not to change this correction in order to retain comparability with other results.

Figures $4 \mathrm{a}$ and $4 \mathrm{~b}$ show the same distributions as Figure 3, after corrections for Coulomb effects and non- $\pi \pi$ pairs. The results of the fits in Figures $4 \mathrm{a}$ and $4 \mathrm{~b}$, in which form (2)

${ }^{\ddagger}$ The jet axis was defined by the sum of the momenta of the charged tracks. 


\begin{tabular}{|c|c|c|r|}
\hline & $r(\mathrm{fm})$ & $\lambda$ & Ref. sample \\
\hline \hline$(a)$ & $\mathbf{0 . 8 3} \pm \mathbf{0 . 0 3}$ & $\mathbf{0 . 3 1} \pm \mathbf{0 . 0 2}$ & +- \\
\hline & $\mathbf{0 . 4 7 \pm 0 . 0 3}$ & $\mathbf{0 . 2 4} \pm \mathbf{0 . 0 2}$ & mixed \\
\hline$(b)$ & $\mathbf{0 . 8 2} \pm \mathbf{0 . 0 3}$ & $\mathbf{0 . 4 5} \pm \mathbf{0 . 0 2}$ & +- \\
\hline & $\mathbf{0 . 4 2 \pm \mathbf { 0 . 0 4 }}$ & $\mathbf{0 . 3 5} \pm \mathbf{0 . 0 4}$ & mixed \\
\hline
\end{tabular}

Table 1: Results of the fit to the data without (a) and with (b) Coulomb and non- $\pi \pi$ corrections.

is used ${ }^{\S}$, are $r=0.82 \pm 0.03 \mathrm{fm}, \lambda=0.45 \pm 0.02(\chi 2=89 / 73 \mathrm{DF}, C(r \lambda)=0.38)$ and $r=0.42 \pm 0.04 \mathrm{fm}, \lambda=0.35 \pm 0.04(\chi 2=155 / 93 \mathrm{DF}, C(r \lambda)=0.55)$ respectively.

The effect of the Coulomb correction is to increase $\lambda$ by about $20 \%$ when the unlikesign reference sample is used and by about $10 \%$ when the mixed reference sample is used. The radius $r$ is only slightly affected by the correction.

The superimposed curves show the results of the fit to these distributions of the expression (2). The results of the fit are presented in Table 1 for the two reference samples, both before (a) and after (b) corrections. The uncertainty resulting from including or removing from the fit the $K_{S}^{o}$ and $\rho^{o}$ regions for $R_{+-}$is within the statistical error.

The results from the two different reference samples are inconsistent, as already observed in [6]. This is interpreted as a consequence of the presence of correlations that the simulation fails to model precisely. Many different causes can contribute to this:

- The presence of resonances not correctly parametrized in the simulation

- A particle from a secondary source, such as a long lived hadron decay, may not contribute to the observed Bose-Einstein correlation. The data have not been corrected for this effect.

- Final state strong interactions are not taken into account. Calculations based on a simplified model [15] suggest anyway that the strong final state interactions in multipion production largely cancel.

The results obtained from the corrected distributions (including Coulomb corrections) using the two reference samples have been averaged and the systematic uncertainty from the choice of the reference sample has been taken to be one half of the difference between the two separate results. This gives:

$$
\begin{aligned}
& r=0.62 \pm 0.04(\text { stat }) \pm 0.20(\text { syst }) \mathrm{fm} \\
& \lambda=0.40 \pm 0.03(\text { stat }) \pm 0.05(\text { syst }) .
\end{aligned}
$$

\footnotetext{
$\S$ The region from $\mathrm{Q}=0.4 \mathrm{GeV} / \mathrm{c}$ to $\mathrm{Q}=0.8 \mathrm{GeV} / \mathrm{c}$ was removed from the fit to figure $4 \mathrm{a}$.

IIn particular, the $\eta^{\prime}$ decay chain $\eta^{\prime} \rightarrow \eta \pi^{+} \pi^{-}, \eta \rightarrow \pi^{+} \pi^{-} \pi^{\circ}$ affects the same region of small relative momenta as the Bose-Einstein effect [14], and was a-priori indicated as a possible source of major systematics. By using kinematic methods in order to reject these contaminating pairs, it was concluded that the $\eta^{\prime}$ in the data sample does not influence the correlation function significantly.
} 


\section{Conclusions}

Bose-Einstein correlations between like-sign pions produced in the decay of the $\mathrm{Z}^{0}$ have been studied with the DELPHI detector at LEP, using a sample of about 76,000 selected hadronic events. The results show a Bose-Einstein enhancement which can be parametrized by the expression $R(Q)=1+\lambda e^{-r^{2} Q^{2}}$, where $\lambda=0.40 \pm 0.03$ (stat) \pm 0.05 (syst) and $r=0.62 \pm 0.04$ (stat) $\pm 0.20($ syst $) \mathrm{fm}$. The large systematic errors come mainly from an inconsistency between the results obtained with two different reference samples.

The full event sample and a subsample of 2-jet events display the same effect when using pairs of opposite sign particles as a reference. This supports the conclusion [16] that the enhancement does not depend on the event topology.

The DELPHI results are comparable with those obtained at lower energy $e^{+} e^{-}$experiments [4]. The measurement of the radius of the interaction region agrees with the recent measurements from the OPAL [5] and ALEPH [6] experiments at LEP. The strength of the effect measured is somehow lower than that published by ALEPH, and considerably lower than that published by OPAL.

\section{Acknowledgments}

We are greatly indebted to our technical staffs and collaborators and funding agencies for their support in building the DELPHI detector and to the many members of the CERN-SL division for the excellent performance of the LEP machine.

We thank A. Giovannini, E. Menichetti and T. Sjöstrand for useful discussions. 


\section{References}

[1] G. Goldhaber et al., Phys. Rev. 120 (1960) 300.

[2] G.I. Kopylov and M.I. Podgoretskii, Sov. J. Nucl. Phys. 19 (1974) 215; G.I. Kopylov, Phys. Lett. 50B (1974) 472.

G. Cocconi, Phys. Lett. 49B (1974) 459.

M. Deutschmann et al., Nucl. Phys. 204B (1982) 333.

A. Giovannini and G. Veneziano, Nucl Phys. B130 (1977) 61.

M. G. Bowler, Z. Phys. C29 (1985) 617.

[3] B. Lörstad, Int. J. Mod. Phys. A4 (1989) 2861.

W. A. Zajc, in "Hadronic Multiparticle Production", P. Carruthers ed., World Scientific (1988) 235.

[4] H. Aihara et al., (TPC-PEP4 Collaboration), Phys. Rev. D31 (1985) 996.

P. Avery et al., (CLEO Collaboration) Phys. Rev. D32 (1985) 2294.

I. Juricic et al (Mark II Collaboration) Phys. Rev D39 (1989) 1.

M. Althoff et al.(TASSO Collaboration), Z. Phys. C30 (1986) 355.

R. C. Walker (AMY Collaboration), UR-1176 (1990).

[5] P.D. Acton et al. (OPAL Collaboration), Phys. Lett. 267B (1991) 143.

[6] D. Decamp et al. (ALEPH Collaboration), CERN-PPE 91/183.

[7] P. Aarnio et al. (DELPHI Collaboration), Nucl. Instr. and Meth. A303 (1991) 233.

[8] P. Aarnio et al. (DELPHI Collaboration), Phys. Lett. 240B (1990) 271.

[9] DELSIM User Manual, DELPHI 87-96 PROG-99, Geneva, July 1989.

DELSIM Reference Manual, DELPHI 87-98 PROG-100, Geneva, July 1989.

[10] T. Sjöstrand, Comp. Phys. Comm. 27 (1982) 243, ibid. 28 (1983) 229. T. Sjöstrand and M. Bengtsson, Comp. Phys. Comm. 43 (1987) 367.

[11] DELPHI Collaboration, "Comparison of data with QCD models", contributed paper to the LP-EPS conference, Geneva, August 1991.

[12] M. Gyulassy, S.K. Kaufmann and L.W. Wilson, Phys. Rev. C20 (1979) 2267.

[13] M. G. Bowler, OUNP-91-23 (1991).

[14] K. Kulka and B. Lörstad, Nucl. Instr. and Meth. A295 (1990) 443.

[15] M. G. Bowler, Z. Phys. C39 (1988) 81.

[16] T. Akesson et al. (AFS Collaboration), Z. Phys. C36 (1987) 517.

M. Althoff et al. (TASSO Collaboration), Z. Phys. C30 (1986) 355.

\section{Figure Captions}

1. Pairs of like-sign (circles) and unlike-sign (asterisks) tracks, as a function of the 4-momentum difference $Q$.

2. Ratio $R_{+-}$between like and unlike sign pairs, for data (circles) and Monte Carlo (asterisks), as a function of the 4-momentum difference $Q$.

3. Ratios (a) $R_{+- \text {,data }} / R_{+-, \mathrm{MC}}$ and (b) $R_{\text {mix,data }} / R_{\text {mix }, \mathrm{MC}}$ (see text) as a function of $\mathrm{Q}$. The best fit to (2) is superimposed as a solid line.

4. Same as Figure 3, after corrections for Coulomb effects and non- $\pi \pi$ pairs. 
Figure 1

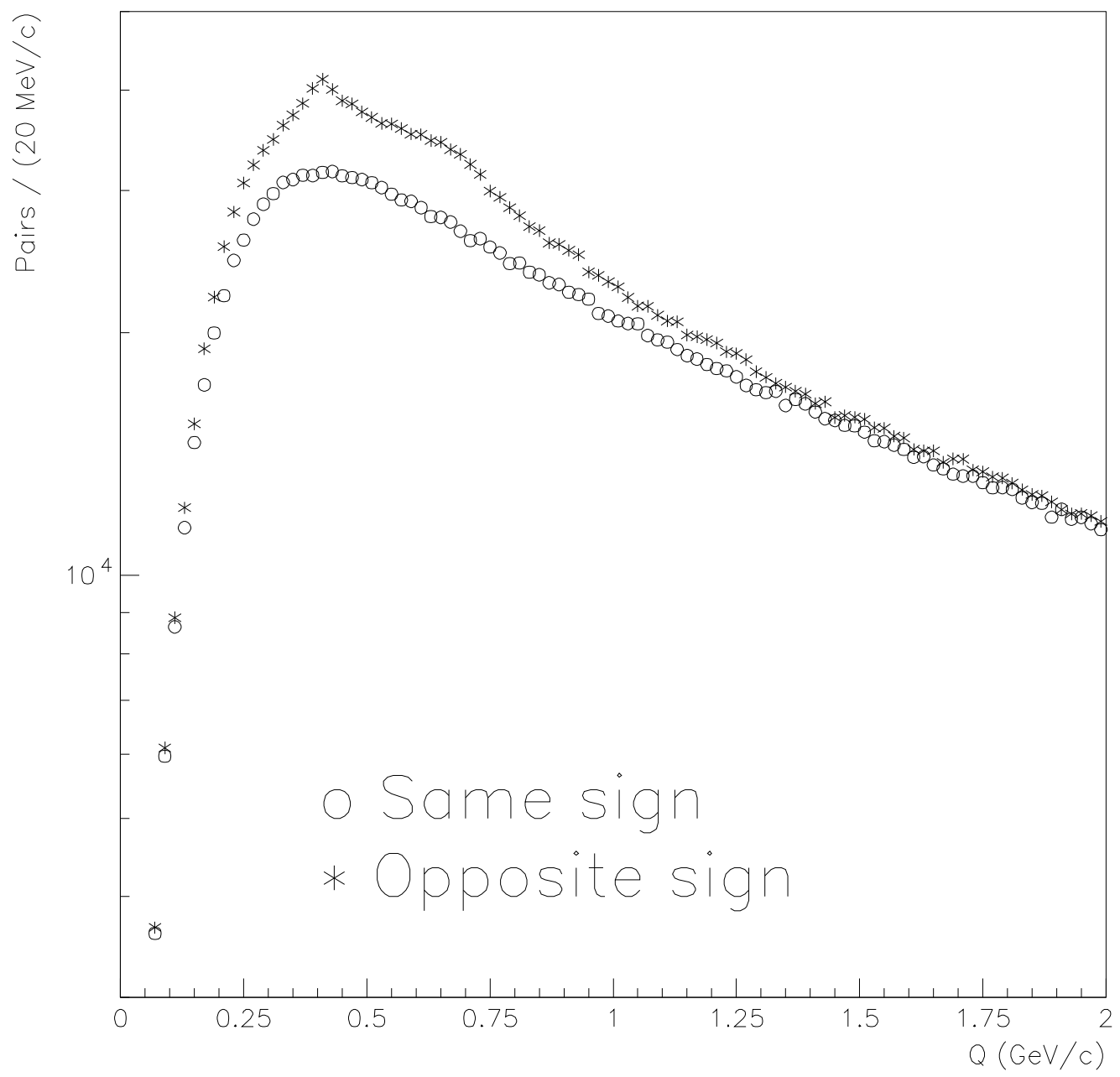


Figure 2

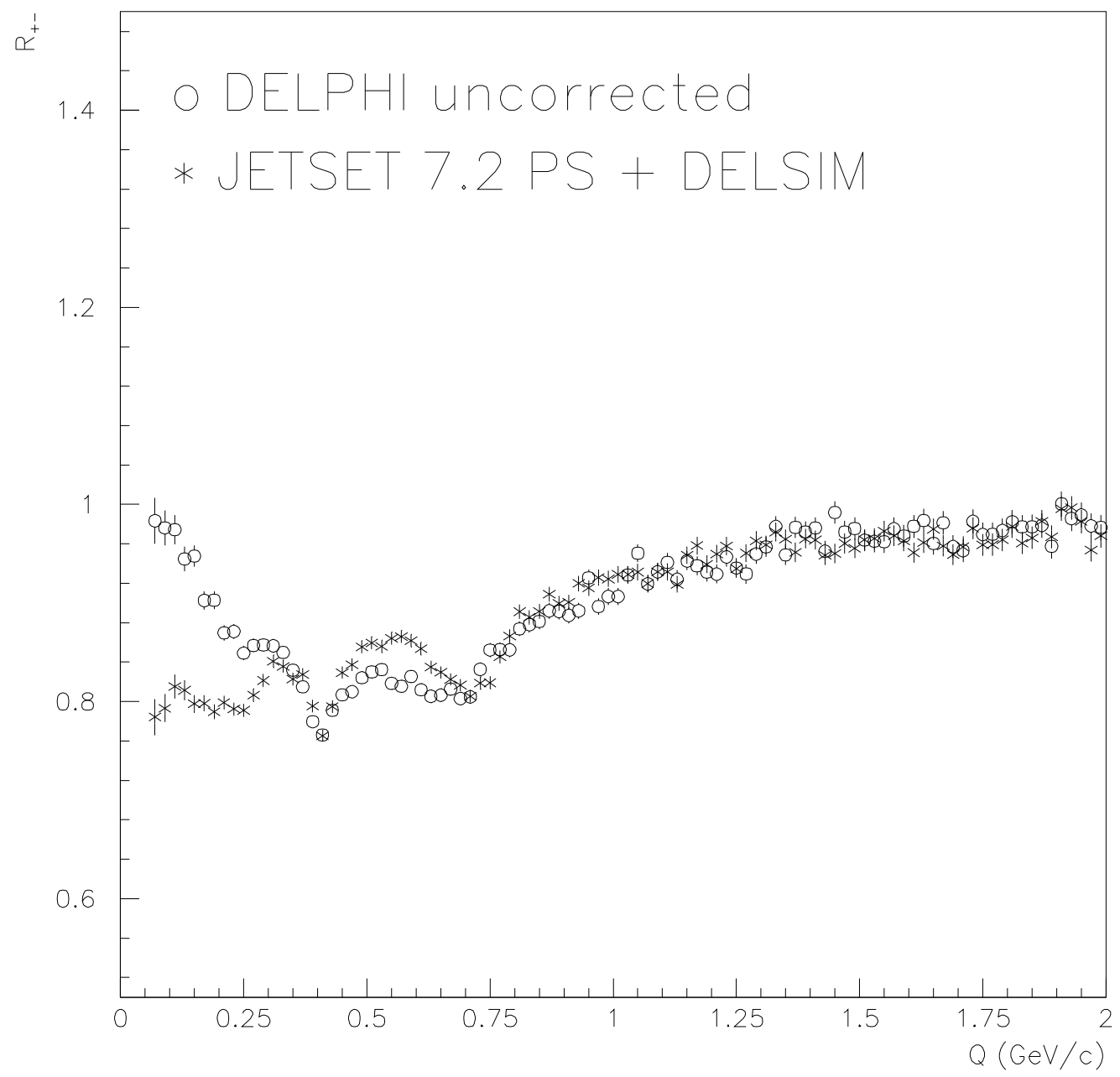


Figure 3
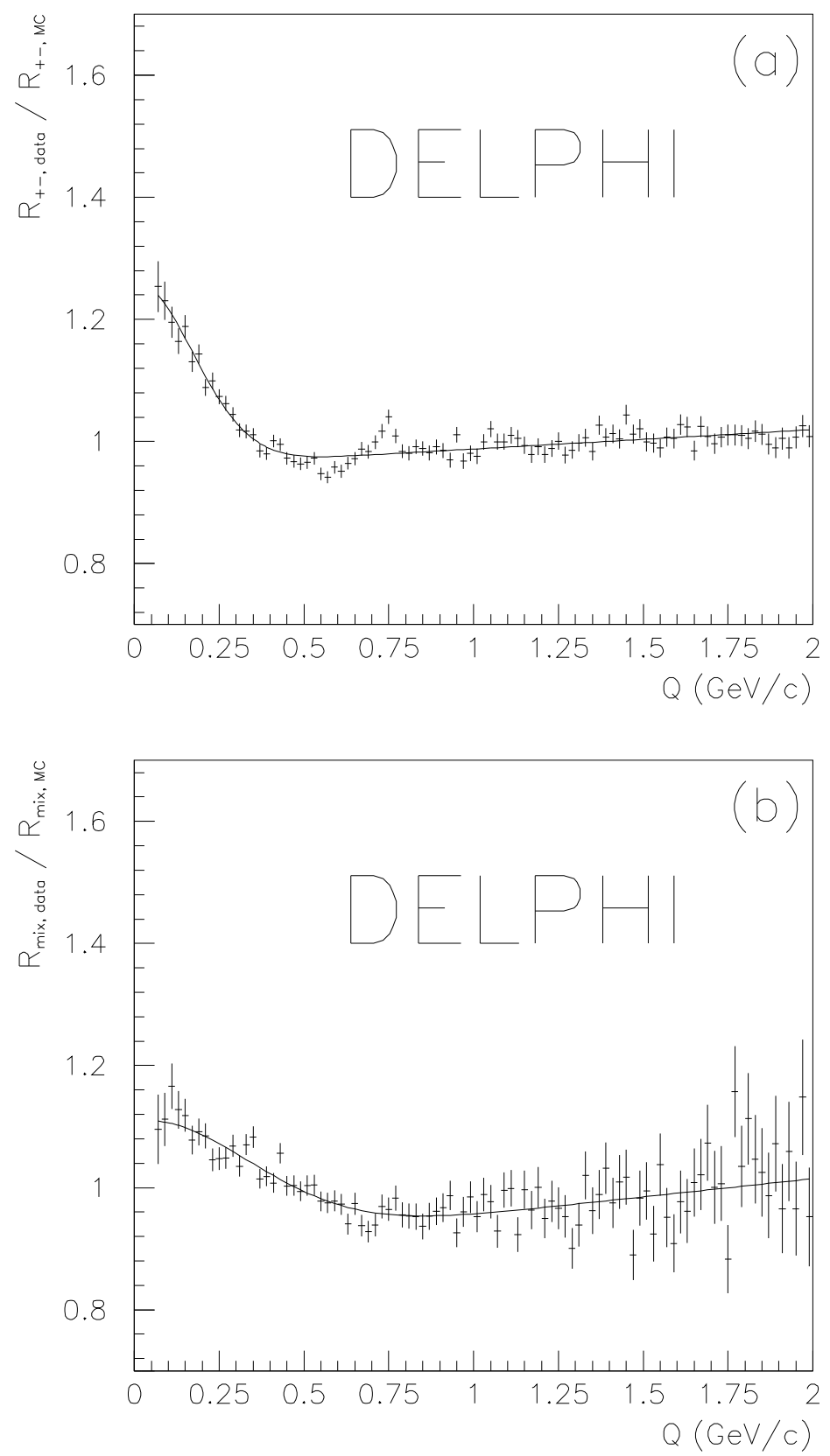
Figure 4
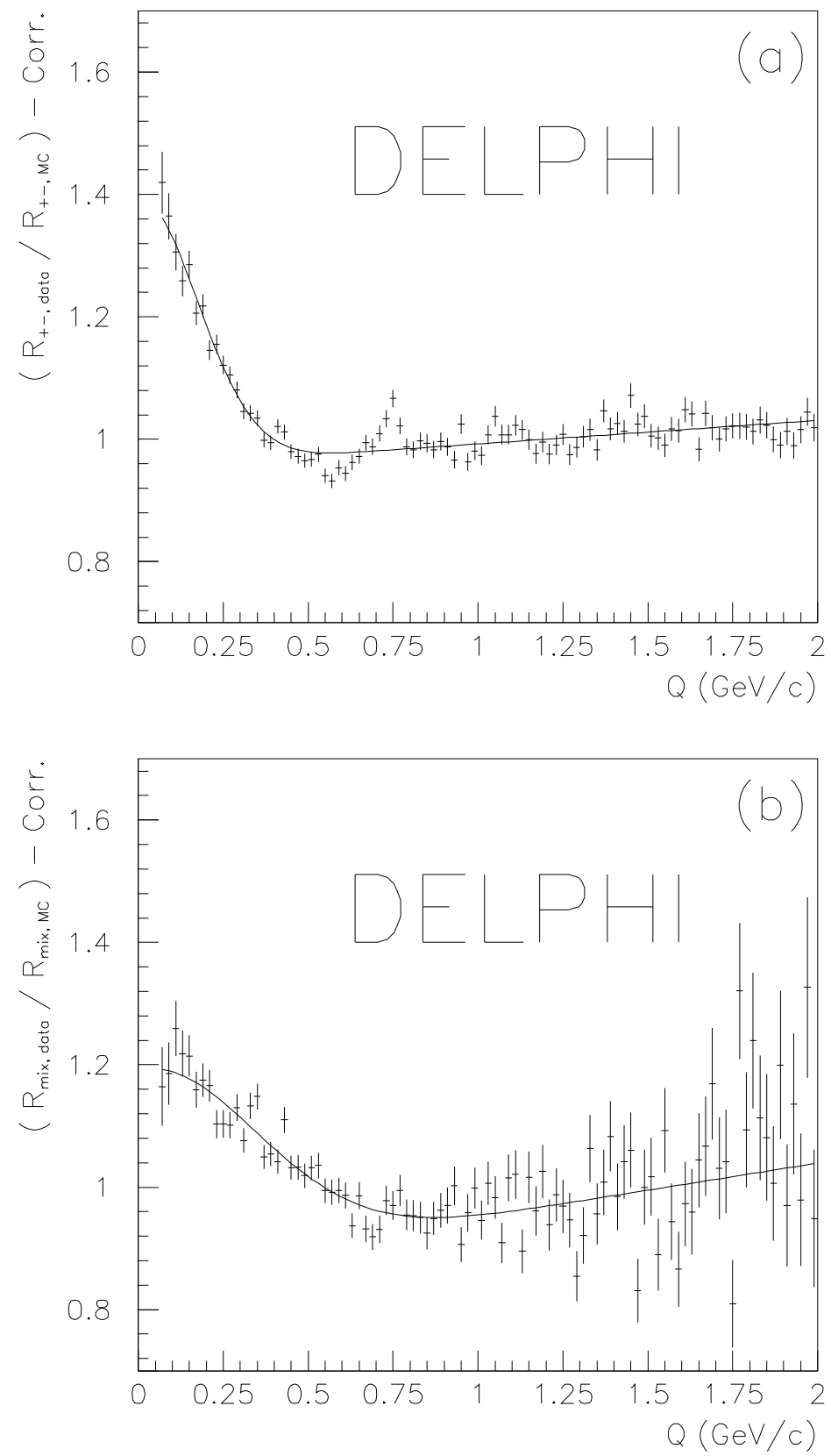